\title{
Reflexionen zur Passung zwischen Praxis und Forschung
}

\author{
Maria Teresa Diez Grieser \\ Psychotherapie-Wissenschaft 9 (2) 87-91 2019 \\ www.psychotherapie-wissenschaft.info \\ CC BY-NC-ND \\ https://doi.org/10.30820/1664-9583-2019-2-87
}

\begin{abstract}
Zusammenfassung: Der Beitrag thematisiert die unterschiedlichen Perspektiven und Bedürfnisse von Forschung und Praxis. Der Austausch zwischen den beiden Systemen wird durch verschiedene Barrieren zusätzlich erschwert. Folgen dieser mangelnden Passung sind auf der einen Seite eine verzögerte und häufig inkohärente Rezeption der Forschungsergebnisse durch die Praxis. Auf der anderen Seite verpasst die Forschung aufgrund ihres Top-down-Vorgehens die Möglichkeit, Praxiswissen im Rahmen zirkulärer Prozesse einzubauen, die zu praxisrelevanten und innovativen Ergebnissen führen könnten. Anhand der Ergebnisse zur desorganisierten Bindungsforschung und zu Umfeldfaktoren bei Traumafolgestörungen werden einzelne Probleme illustriert.
\end{abstract}

Schlüsselwörter: Forschung-Praxis-Unterschiede, Rezeption von Forschungsergebnissen, desorganisierte Bindung, Umfeldfaktoren

Praxis und Theorie sind zwei Begriffe, in deren Verhältnis zueinander häufig ein Widerspruch vermutet wird, obwohl sie tatsächlich in einem rationalen Verhältnis zueinander stehen.

Forschung und Praxis haben unterschiedliche Referenzsysteme, was ihren Austausch und ihre Kooperation negativ beeinflussen kann. Dieses als science-practice gap bekannte Phänomen führt dazu, dass Forschungsergebnisse zeitlich verzögert oder nur teilweise in der Praxis Eingang finden. Gründe dafür sind auf der einen Seite das geringe Interesse von Forschenden an Praxiswissen, was zu Top-down-Forschungsergebnissen führt, die von den Praktizierenden in Bezug auf ihre Praxisrelevanz kritisch betrachtet werden (Bohus, 2015). Auf der anderen Seite ist der Umgang der Praxis mit relevanten Forschungsergebnissen häufig inkohärent. Dies ist teilweise durch identifizierbare Barrieren bedingt, die durch einen intensiveren Austausch zwischen den beiden Systemen abgebaut werden müssten. Der Aufbau von Netzwerken, die aus Forschenden und Praktizierenden bestehen, kann eine mögliche Lösungsstrategie darstellen (Tasca et al., 2014), die Implementierung solcher Strukturen findet aufgrund fehlender finanzieller Ressourcen jedoch selten statt. Eine wichtige Barriere, die den Austausch und die Zusammenarbeit zwischen Forschung und Praxis erschwert, bleibt die unterschiedliche Kultur, die sich insbesondere im Bereich der Güte- bzw. Qualitätskriterien zeigt. Für die Forschung sind bekanntermassen die vier Gütekriterien der Objektivität, der Repräsentativität, der Validität und der Reliabilität massgeblich (Bortz, 2005). Im Gegensatz dazu sind für die Praxis Kriterien zentral, die die Frage nach der passenden Intervention für die jeweilige Problemlage beschreiben und sich dabei einerseits auf Forschungsergebnisse, andererseits aber auf best practice-Beispiele beziehen. Um diese unterschiedlichen
Perspektiven und Bedürfnisse konstruktiv zu nutzen, braucht es bei der Erarbeitung von Fragestellungen, bei der Auswahl der Messmethoden und der Stichproben zirkuläre Prozesse, die durch Akzeptanz auf beiden Seiten und Umsetzbarkeit in der Praxis charakterisiert sind.

Strukturierte Kooperationen können Vorteile für Forschung und Praxis bringen: Die Forschung kann ihre durch Methodenzwang geprägte Sicht auf die Praxis revidieren und angewandte Forschung betreiben, während die Praxis disziplinierter Phänomene und Prozesse beobachten und in wissenschaftliche Fragestellungen fassen muss (Bohus, 2015).

Im letzten Jahrzehnt hat sich das Bedürfnis der Praxis nach mehr Anwendbarkeit der Theorie und Umsetzung der Forschungsergebnisse verstärkt. Dahinter steht die Ernüchterung über den Ertrag und die praktische Ergiebigkeit der empirisch analytischen Forschung, vor allem ihr Mangel an praktischer Relevanz. Des Weiteren führt der Wunsch der Forschung nach möglichst viel Datenmaterial häufig zu einer schier unüberschaubaren Komplexität der Auswertung, die letztlich auch auf der Interpretationsebene multideterminiert bleibt, was der Praxis kaum neue Erkenntnisse bringen kann. Verstärkt wird dieses Phänomen zusätzlich durch Forschungsstrategien, die hypothesen- oder sogar theoriefrei messen möchten. Dahinter lässt sich eine Haltung vermuten, die in etwa mit folgendem Satz umschrieben werden könnte: "Wenn man Alles misst, wird man schon etwas sagen können.» Was dann schliesslich gesagt wird, bringt selten neue Erkenntnisse, sondern dient häufig der Validierung bereits vorhandenen praktischen Wissens. Einen wichtigen Platz für die Praxis hat die Forschung jedoch dort, wo die Experimente und Studien einen empirischen Hintergrund für deren theoretische Annahmen bieten. Nicht wenige Forschungsergebnisse werden von Praktizierenden mit 
Begeisterung aufgenommen und unkritisch in ihre Arbeit integriert. Andere Forschungsergebnisse werden jedoch kaum wahrgenommen oder weitgehend ignoriert und die «bewährte» Praxis wird weitergeführt.

Im vorliegenden Beitrag soll anhand zweier Beispiele, die mit Bindungstheorie und Traumafolgestörungen zwei relevante und bekannte Themen betreffen, illustriert werden, wie Forschungsergebnisse in der Praxis rezipiert werden.

\section{Zwei Beispiele}

\section{Diskurs um desorganisierte Bindung}

Die von Bowlby (1969) begründete Bindungstheorie hat sowohl im Bereich der psychoanalytischen Kinder- als auch der Erwachsenenpsychotherapie eine grosse Bedeutung erlangt. Insbesondere seitdem die Bindungsforschung die Verhaltens- um die Repräsentationsebene erweitert hat, ist sie zu einer wichtigen ergänzenden Erkenntnisquelle für die therapeutische Arbeit geworden, da die Berücksichtigung der spezifischen Beziehungsmuster der PatientInnen sowie insbesondere die Handhabung der Übertragung dadurch verbessert worden ist. Die von Ainsworth und KollegInnen (1978) beschriebenen Bindungsstile, die jedes Kind im Laufe der ersten beiden Lebensjahre zu bedeutsamen Bezugspersonen aufbaut, haben das Verständnis für die menschlichen Grundbedürfnisse geschärft und spielen als Hintergrundwissen für die Gestaltung von entwicklungs- und resilienzfördernden Bedingungen, Interventionen und Angeboten eine herausragende Rolle. Die von Main und Solomon (1986) zu einem späteren Zeitpunkt erfasste desorganisierte Bindung hat die Palette der möglichen Bindungsstile entscheidend erweitert und den Blick für die diesem Bindungstyp zugrundeliegenden Bindungserfahrungen geschärft. Die fruchtbare Entwicklung der Theorie des Mentalisierens hat ausserdem im letzten Jahrzehnt als umbrella concept Forschungsbefunde und Erkenntnisse aus der Psychoanalyse, der Bindungstheorie, der Entwicklungspsychologie und der Neurobiologie miteinander verbunden und der Theorie, der Forschung und der Praxeologie der Psychotherapie zentrale Impulse, neue Sichtweisen und neue konkrete Vorgehensweisen ermöglicht (Diez Grieser \& Müller, 2018).

Die aus der Bindungstheorie und -forschung stammenden Befunde zeichnen sich durch unmittelbare Nachvollziehbarkeit aus, was ihre schnelle Rezeption und Implementierung in der Praxis erklärt. Die Anwendung der Bindungstheorie im Kinder- und Jugendbereich sowohl in klinischen als auch in pädagogischen Kontexten zeigt jedoch besonders deutlich, welche Diskrepanz zwischen der Bindungstheorie und der Forschung auf der einen Seite und den Anwendungen in der Praxis auf der anderen Seite bestehen kann. Insbesondere die notwendige Unterscheidung zwischen Bindungsstilen-/typen und Bindungsstörungen findet in der Praxis zu wenig statt, sodass es zu eigentlichen Fehlanwendungen kommen kann, die negativ auf betroffene Kinder und ihre Familien einwirken können.

In Anlehnung an den Artikel von Granqvist und KollegInnen (2017), der von sämtlichen aktuell relevanten BindungstheoretikerInnen und -forscherInnen mitgeschrieben worden ist, soll im vorliegenden Beitrag auf die Rezeption und Handhabung der desorganisierten Bindung im klinischen Kontext und bei politischen Entscheidungsträgern hingewiesen werden. An diesem Beispiel zeigt sich mit besonderer Prägnanz, wie ein theoretisches Konzept und entsprechende Forschungsergebnisse «falsch» interpretiert und angewendet werden. Die AutorInnen erörtern den Erkenntnisstand bezüglich desorganisierter Bindung mittels zehn Thesen, die im Folgenden zusammengefasst und übersetzt wiedergegeben werden.

1. Die Kategorie der desorganisierten Bindung kann durch trainierte Kodierer kindlichem Verhalten (Alter: 12-20 Monate) in der Fremden-Situation zugeordnet werden, wenn es eine ausreichende Übereinstimmung mit einem oder mehreren der sieben von Main und Solomon (1986, 1990) beschriebenen Dimensionen und aufgeführten Verhaltensweisen gibt.

2. Die von Main und Solomon beschrieben Verhaltensweisen können aus sehr unterschiedlichen Gründen auftreten. Auf einem niedrigen Niveau treten sie häufig bei Kindern in belasteten Lebens- und Familiensituationen auf. Lediglich wenn die Verhaltensweisen eine hohe Intensität haben, können diese als desorganisierte Bindung klassifiziert werden.

3. Desorganisierte Bindung kommt bei misshandelten Kindern häufiger vor, das Vorkommen weist aber nicht unbedingt auf Misshandlung hin, das heisst, dass das Vorhandensein einer desorganisierten Bindung nicht zur Diagnose einer Misshandlung verwendet werden kann.

4. Es gibt verschiedene Pfade, die zu desorganisierter Bindung führen können wie bspw. unverarbeitete Traumata der Eltern. Des Weiteren können genetische Aspekte und Temperament eine Rolle spielen.

5. Forschungsergebnisse auf der Gruppenebene zeigen, dass desorganisierte Bindung ein kleiner bis moderater Prädiktor für die Entwicklung von sozialen und Verhaltensproblemen ist. Desorganisierte Bindungen müssen nicht zwangsläufig zu späteren Problemen führen.

6. Desorganisierte Bindung ist nicht eine validierte klinische Diagnose auf individuellem Niveau; dies im Gegensatz zu den Bindungsstörungen, die in den psychiatrischen Klassifikationssystemen beschrieben werden. Diese klinischen Störungen sind in verschiedenen Settings und mit verschiedenen Erwachsenen vorhanden. Im Gegensatz dazu ist eine desorganisierte Bindung keine fixe Eigenschaft eines individuellen Kindes, sondern beziehungsspezifisch.

7. Es haben in den letzten Jahren (z. B. bei Entscheidungen bezüglich Fremdunterbringungen von Kindern) Fehlentscheidungen stattgefunden, die Folge falscher Annahmen bezüglich der desorganisierten Bindung waren (s. Punkte 1 bis 6). 
8. Fehlanwendungen können unterprivilegierte Familien selektiv schädigen, gegen Menschen- und Kinderrechte verstossen und eine diskriminierende Praxis gegen Minderheiten darstellen.

9. Es ist wichtig anzuerkennen, dass wir über eine breitgestützte Evidenz darüber verfügen, dass sowohl bindungsbasierte Interventionen als auch naturalistisch auftretende reparative Erfahrungen (z.B. stabile, sichere und förderliche Beziehungen) intergenerationelle Zyklen von Kindesmisshandlungen unterbrechen können und dazu beitragen, dass der Anteil an Kindern mit desorganisierten Bindungen geringer wird.

10. Der eigentliche praktische Nutzen von Bindungstheorie und -forschung liegt darin, das Verständnis von Familien zu fördern und evidenzbasierte Interventionen anzubieten: "In this way, attachment theory, assessments, and research can have major roles to play in clinical formulation and supportive welfare and clinical work» (Granqvist et al., 2017, S. 2f.).

Der zitierte Artikel ist insofern für das Thema der Passung zwischen Forschung und Praxis von besonderem Interesse als hier seitens der Forschenden und TheoretikerInnen sehr klar und praxisbezogen die Chancen, aber insbesondere auch die Grenzen des Phänomens verdeutlicht werden, sodass Fehlinterpretationen im praktischen Feld minimiert werden können. Dies ist allerdings erst im Nachgang geschehen, nachdem die Missverständnisse und die Fehlanwendungen im Feld bereits seit einiger Zeit weit verbreitet sind.

An diesem Beispiel zeigt sich, wie die Praxis Ergebnisse aus der Forschung, vor allem wenn sie eine hohe Plausibilität aufweisen und mit den Perspektiven der Praktizierenden gut vereinbar sind, breit rezipiert und implementiert, ohne die Grenzen für die Umsetzung der Ergebnisse in der Praxis zu erkennen.

Es ist einerseits wünschenswert, dass Professionelle verschiedener Praxisbereiche in ihren Grundausbildungen sowie in den späteren Spezialisierungen Wissen und Kompetenzen im Zusammenhang mit dem Verstehen und Einordnen von Forschungsresultaten erwerben. Andererseits stellt sich die Frage, wie die Forschung und die Forschenden stärker in den Blick bekommen, dass ihre Tätigkeit und die damit verbundenen Ergebnisse auf differenzierte und für die Praxis klare Art und Weise vermittelt werden müssen/sollen. In den letzten Jahren findet sich in vielen Fachzeitschriften die Rubrik «Fazit für die Praxis», die ein Versuch darstellt, Ergebnisse bezüglich Anwendbarkeit und praktischer Relevanz für Praktizierende zusammenfassend zu erörtern. Es ist jedoch auch zu fordern, dass Forschende - wie beim zitierten Artikel - den Umgang mit Forschungsbefunden im Feld besser begleiten und ihre Verantwortung als WissenschaftskommunikatorInnen besser wahrnehmen. Es ist sicherlich kein einfaches Unterfangen, wenn es - wie bei den Ergebnissen der Bindungstheorie - um einen fast unüberschaubaren Korpus von Wissen geht, der einiger Anstrengung bedarf, damit brauchbare und klare Botschaften an die Praxis gehen können.

\section{Diskurs um Folgen schwerer Belastungen}

Ein weiteres Beispiel soll eine andere Diskrepanz zwischen Forschung und Praxis darstellen und zu weiterer Reflexion anregen. Anhand dieses Beispiels soll veranschaulicht werden, dass es nicht wie beim zuvor erwähnten Beispiel um eine zu starke und konkretistische Übernahme von Forschungsergebnissen geht, sondern um die ungenügende beziehungsweise inkohärente Aufnahme von Erkenntnissen und Befunden durch Praktizierende.

Im Bereich der Psychotraumatologie hat in den letzten zwei Jahrzehnten eine enorme Entwicklung stattgefunden: Traumawirkungen, Trauma- und Resilienzprozesse können festgestellt werden und entsprechende Hilfestellungen sowohl im therapeutischen als auch im sozialen Feld sind entwickelt und implementiert worden. Nicht zu Unrecht wird in der Praxis zunehmend beklagt, dass kritische Lebensereignisse und belastende Erfahrungen häufig vorschnell mit Traumatisierungen gleichgesetzt werden, das heisst, dass eine Ausweitung und Aushöhlung der Traumadefinition stattfindet. Inwiefern aus einer potenziell traumatischen Erfahrung eine Traumafolgestörung wird, ist von verschiedenen Faktoren abhängig, die je nach Ausprägung und Intensität die Wirkung der belastenden Erfahrung abpuffern können. Diese prä-, peri- und posttraumatischen Risiko- und Schutzfaktoren können - wie die psychotraumatologische Forschung der letzten 70 Jahre zeigt - abhängig von ihrer Wechselwirkung zu unterschiedlichen Resultaten führen (Zeidner \& Aharoni-David, 2014).

Wie Held (2019, S. 111f.) in einem kürzlich erschienenen Artikel festhält, sind auch «bei den stärksten traumatischen Einwirkungen» lediglich bei weniger als $50 \%$ Traumafolgestörungen feststellbar. Er stellt Forschungsdaten aus den Untersuchungen mit Holocaust-Überlebenden ins Zentrum seiner Ausführungen, weil es «keine auch nur annähernd vergleichbar gute Daten gibt» (ebd., S. 113). Es ist deshalb bemerkenswert, dass die Befunde aus diesen Studien, die kohärente Ergebnisse aufweisen, relativ wenig rezipiert werden und viele Forschungsarbeiten in diesem Feld sich mit basalen Fragestellungen beschäftigen, die bereits beantwortet sind.

Wie es dazu kommt, dass trotz schwerer Belastungen und potenziell traumatisierender Erfahrungen gute Entwicklungen und ein gelingendes Leben möglich sind, wird durch die Resilienzforschung sowie durch Konzepte, die Umfeldressourcen in den Blick nehmen, untersucht und teilweise deutlich beantwortet. Die Forschungsergebnisse aus diesem Bereich konnten zeigen, dass die Unterstützung durch Bezugspersonen sowie die gesellschaftliche Anerkennung und Teilhabe betroffener Menschen die Chancen für positive Entwicklungen bei Traumatisierungen erhöhen. Für die Praxis bedeutet dies, dass bei der Begleitung und Behandlung traumatisierter Menschen die Erfassung und Bereitstellung unterstützender Umfeldfaktoren und Ressourcen ein Schwerpunkt sein sollte. Dennoch zeigt sich, dass psychotraumatologische Behandlungen verschiedener Psychotherapieschulen den Fokus auf die Traumakonfrontation und -verarbeitung im Rahmen 
von Psychotherapien legen und wichtige salutogenetische Faktoren und eine Ressourcenaktivierung vernachlässigen (Reddemann \& Wöller, 2017).

Des Weiteren verweist Held (2019, S. 128) in seiner Arbeit darauf, dass die referierten Forschungsergebnisse PsychotherapeutInnen zeigen können, dass diese im Rahmen der Hilfestellungen für traumatisierte Menschen zwar eine wichtige, nicht aber eine dominierende Rolle einnehmen können.

\section{Abschliessende Bemerkungen}

Forschung und Praxis bedingen einander, um ihren gemeinsamen Gegenstand zu erfassen, abzubilden und weiterzuentwickeln. Insbesondere die Praxis ist auf eine Forschung angewiesen, die Phänomene differenziert beschreiben und einen Beitrag dazu leisten kann, dass in der Praxis Handlungsmuster und Sichtweisen besser reflektiert werden können.

Die Weiterentwicklung der Forschung in den letzten Jahrzehnten hat zu einer besseren Passung bezüglich der Praxisanliegen geführt, weil das Methodenrepertoire um qualitative und prozessbezogene Ansätze erweitert worden ist (Kelle, 2014). Praktizierende haben ihrerseits die Erfahrung gemacht, dass es zielführender für sie ist, wenn sie in Forschungsprozessen von Anfang an mitarbeiten. Es zeigt sich aber immer wieder, dass Forschung und Praxis unterschiedlichen Funktionsmustern folgen und dadurch die Kommunikation zwischen diesen beiden Welten erschwert wird.

Beide Beispiele verdeutlichen, dass es der Forschung häufig nicht gelingt, ihre Ergebnisse so aufzubereiten, dass Praktizierende deren Validität und die Grenzen der Anwendung in der Praxis nachvollziehen können. Praktizierende ihrerseits haben entweder zu wenig zeitliche Ressourcen für eine vertiefte Auseinandersetzung mit Forschungsthemen und/oder zu wenig Wissen, um die Befunde in ihrer praktischen Relevanz und Umsetzbarkeit einzuordnen. Es scheint offensichtlich, dass etwas Drittes gebraucht wird, das zwischen beiden Welten vermitteln kann. Während die Wissenschaftskommunikation mittlerweile als Brücke zwischen Wissenschaft und Öffentlichkeit eine wichtige Funktion übernimmt und in vielen Organisationen eine Selbstverständlichkeit ist, bleibt die Kommunikation zwischen Forschung und Praxis beispielsweise in der Psychotherapieforschung Sache der einzelnen Fachpersonen. Die Vermittlung von Forschungsergebnissen sowie Hilfestellungen bezüglich deren Einschätzung und praktischer Relevanz wird zwar immer wichtiger und zunehmend Teil der Fortbildungscurricula, trotzdem sind viele Praktizierende nach wie vor skeptisch und vertrauen lieber ihrer Intuition und persönlichen Erfahrung als Forschungsergebnissen.

Noch immer arbeiten Forschung und Praxis oftmals gegen- anstatt miteinander, was sich schlussendlich negativ auf die Forschung, aber auch auf die Praxis auswirkt. Der vorliegende Artikel versuchte, die Probleme zu benennen, die entstehen, wenn Forschungsergebnisse von Prakti- zierenden falsch interpretiert oder ignoriert werden und umgekehrt Forschende nicht bemüht sind, ihre Ergebnisse praxisnah zu vermitteln.

\section{Literatur}

Bohus, M. (2015). Elfenbeintürme im Treibsand oder: Was macht es so schwierig, Erkenntnisse aus der Forschung in der therapeutischen Praxis umzusetzen? Verhaltenstherapie, 25, 145-155. https://doi.org/ $10.1159 / 000381154$

Bortz, J. (2005). Statistik für Human- und Sozialwissenschaftler. 6. Aufl. Berlin: Springer.

Bowlby, J. (1969). Attachment and Loss, Vol. 1: Attachment. London: Hogarth.

Ainsworth, M.D.S., Blehar, M.C., Waters, E. \& Wall, S. (1978). Patterns of Attachment: A psychological Study oft he Strange Situation. Hillsdale/NY: Erlbaum.

Diez Grieser, M.T. \& Müller, R. (2018). Mentalisieren mit Kinder und Jugendlichen. Stuttgart: Klett-Cotta.

Granqvist, P., Sroufe, A., Dozier, M., Hesse, E., Steele, M., van Ijzendoorn, M., Solomon, J., Schuengel, C., Fearon, P., Bakersmans-Kranenburg, M., Steele, H., Cassidy, J., Carlson, E., Madigan, S., Jacobvitz, D., Foster, S., Behrens, K., Rifkin-Graboi, A., Gribneau, N., Spangler, G., Ward, M. J., True, M., Spieker, S., Reijman, S., Reisz, S., Tharner, A., Nkara, F., Goldwyn, R., Sroufe, J., Pederson, D., Pederson, D., Weigand, R., Siegel, D., Dazzi, N., Bernard, K., Fonagy, P., Waters, E., Toth, S., Cicchetti, D., Zeanah, C.H., Lyons-Ruth, K., Main, M. \& Duschinsky, R. (2017). Disorganized attachment in infancy: a review oft he phenomenon and ist implications for clinicians and policy-makers. Attachment \& Human Development, 1-25. https://doi.org/10.1080/14616734.2 017.1354040

Held, T. (2019). Die potentiell traumatische Erfahrung - wie entscheidet sich, ob es zur Traumafolgestörung kommt? Kinderanalyse, 27(2), 111-132.

Kelle, U. (2014). Mixed Methods. In N. Baur \& J. Blasius (Hrsg.), Handbuch Methoden der empirischen Sozialforschung (S. 153-166). Wiesbaden: Springer.

Main, M. \& Solomon, J. (1986). Discovery of an insecure disorganized/disoriented attachment pattern. In T. B. Brazelton \& M. W. Yogman (Hrsg.), Affective Development in Infancy (S. 95124). Norwood: Ablex.

Main, M. \& Solomon, J. (1990). Procedures for identifying infants as disorganised/disoriented during the Ainsworth Strange Situation. In M.T. Greenberg, D. Cicchetti \& E. M. Cummings (Hrsg.), Attachment in the preschool years (S. 121-160). Chicago/IL: University of Chicago Press.

Reddemann, L. \& Wöller, W. (2017). Komplexe Posttraumatische Belastungsstörung. Göttingen: Hogrefe.

Tasca, G. A., Grenon, R., Fortin-Langenier, B. \& Chyurlia, L. (2014). Addressing Challenges and Barriers to Translating Psychotherapy Reserach into Clinical Practice: The Development of a Psychotherapy Practice Research Network in Canada. Canadian Psychology, 55(3), 197-203. https://doi.org/10.1037/a0037277

Zeidner, M. \& Aharoni-David, E. (2014). Memories of Holocaust-related traumatic experiences, sense of coherence, and survivors'subjective well-being in late life: some puzzling findings. Anxiety, Stress and Coping, 28(3), 254-271. https://doi.org/10.1037/a003727710. 1080/10615806.2014.954244

\section{Reflections on the fit between practice and research}

Abstract: The paper discusses the different perspectives and needs of research and practice. The exchange between the two systems is made even more difficult due to various barriers. The consequences of this bad fit are a delayed and frequently 
incoherent reception of the research results by the practical field. On the other hand, due to its top-down approach, research misses opportunities to integrate practical knowledge in circular processes that could lead to practice-relevant and innovative results. Individual problems are illustrated based on the results on disorganized attachment research and on environmental factors in post-traumatic stress disorders.

Key words: research-practice differences, reception of research results, disorganized attachment, environmental factors

\section{Riflessioni sull'adattamento tra pratica e ricerca}

Riassunto: L'articolo tratta le diverse prospettive e le esigenze di ricerca e pratica. Lo scambio tra i due sistemi è reso ancora più difficile da varie barriere. Le conseguenze di questa mancanza di adattamento sono da un lato una ricezione ritardata e spesso incoerente dei risultati della ricerca da parte della pratica. D'altra parte, a causa del suo approccio top down, la ricerca perde l'opportunità di integrare le conoscenze pratiche nel contesto dei processi circolari, che potrebbero portare a risultati pratici e innovativi. Sulla base dei risultati della ricerca sull'attaccamento disorganizzato e dei fattori ambientali nei disturbi correlati a trauma, sono illustrati singoli problemi.

Parole chiave: differenze ricerca-pratica, ricezione di risultati di ricerca, attaccamento disorganizzato, fattori ambientali

\section{Die Autorin}

Maria Teresa Diez Grieser, Dr., ist Lehrbeauftragte an der Universität Basel und Dozentin am Psychoanalytischen Institut Zürich, an der Hochschule für Soziale Arbeit Luzern und am Schweizerischen Institut für Psychotraumatologie in Winterthur.

\section{Kontakt}

Dr. phil. Maria Teresa Diez Grieser

Gartenhofstrasse 1

CH-8004 Zürich

E-Mail: mtdiez@bluewin.ch 